\title{
Flaubert et la caricature : l'exigence de la modernité
}

Du vivant de Flaubert, quelques rares caricatures du romancier sont publiées, qui ne manquent pas de connaître une notoriété durable. Entre autres : celle d'Eugène Giraud, vers 1866, exécutée lors des "soirées du Louvre » organisées par le comte de Nieuwerkerke, l'ami de la princesse Mathilde, qui jugera d'ailleurs l'expression du visage de l'écrivain bien rendue ${ }^{1}$; celle de Bertall (de son vrai nom : Charles Albert d'Arnoux), qui montre l'écrivain derrière le cochon de saint Antoine ${ }^{2}$; enfin, la plus célèbre assurément, celle de Lemot qui s'en prend au réalisme d'un Flaubert scalpel à la main, en train de disséquer le cour d'Emma ${ }^{3}$. Plaisent-elles à Flaubert ? On ne le sait guère mais l'histoire littéraire a conservé une indication contraire. En 1869, en effet, l'écrivain ne manque pas de s'opposer à une pareille pratique en déniant le droit au caricaturiste André Gill de renouveler l'expérience. Alors que celui-ci, comme le veut la pratique, demandait son consentement à l'écrivain, Flaubert lui répondit:

Monsieur, Je ne puis vous accorder la permission que vous me demandez, l'ayant déjà refusée à plusieurs autres. Je tâche, autant qu'il m’est possible, d'amuser le public par mes livres. C'est bien le moins que je réserve pour moi mon visage! Je suis fâché de vous

Thierry Poyet - maître de conférences HDR à l'Université Clermont Auvergne (Inspe). Adresse de correspondance : Inspe, 36 avenue Jean-Jaures, 63400 Chamalières, France ; e-mail : thierry.poyet@uca.fr

ORCID iD : https://orcid.org/0000-0002-1912-6645

1. Le portrait aurait été reproduit, peut-être pour la première fois, dans Les Maîtres de la Caricature Française au XIX $X^{e}$ siècle, Paris, Quantin, s.d. [1888], puis dans Armand Dayot, Le Second Empire, 18511870, Flammarion, s.d. [1900], p. 227.

2. Elle est publiée dans L'Illustration, 2 mai 1874, p. 289.

3. Elle est publiée dans La Parodie, 5-12 décembre 1869. Ces trois caricatures sont visibles sur le site « Flaubert » de l'Université de Rouen/CÉRÉdI, à l’onglet « Iconographie ». 
désobliger, et je vous prie, Monsieur, d'agréer mes excuses, avec toute l'assurance de ma considération. (Flaubert, Correspondance, avant le 5 décembre 1869, V, p. 1035) ${ }^{4}$

Flaubert se montre-t-il vexé d'avoir été caricaturé ? Voit-il dans le portrait-charge une raillerie pénible, voire une manière de le ridiculiser ? En tout cas, son humour connaît ses limites tout comme la promotion de la liberté artistique.

Le photophobe ${ }^{5}$ Flaubert réussit à échapper à la déferlante caricaturale à une époque où le développement de la presse amplifie au contraire la publication des représentations les plus outrancières. Car, de plus en plus répandue et populaire, la caricature délivre son message non seulement avec simplicité mais encore avec une rapidité qui lui assure une efficacité redoutable. En allant droit au but, en usant d'un manichéisme parfois un peu brutal mais toujours efficace, elle s'impose avec la seconde moitié du XIX ${ }^{\mathrm{e}}$ siècle comme une œuvre artistique de grande consommation. Sa modernité devient incontestable. Comment envisager que le romancier Flaubert, considéré justement par l'histoire littéraire comme l'écrivain de la modernité, refuse de s'abandonner alors à ce qui devient le nouveau genre d'expression en vogue ? Mais y a-t-il un vrai refus de la part du Normand?

Entre un Flaubert caricaturé et un Flaubert caricaturiste, se joue un long combat : celui de la bêtise et de la légitimité, en une affaire politique de la plus haute importance, quand les inclinations nihilistes rencontrent justement le devoir de modernité.

\section{Flaubert caricaturé : de l'incompréhension malheureuse à la bêtise}

La caricature s'apparente souvent à un discours du pauvre. On connaît l'étymologie du mot, issu du latin populaire " caricare » qui signifie charger, exagérer ; pour Flaubert, une caricature se confond trop souvent avec une lecture hâtive, une volonté aveugle de tailler à grands coups de serpe dans la complexité, de réduire à quelques traits outranciers les sillons complexes, mêlés et même embrouillés d'un visage qui a vécu ou d'un caractère aux multiples facettes. L'écrivain le sait parce qu'on n'a pas cessé de le ramener à ses apparences les plus trompeuses.

Celui que Jean-Paul Sartre réduit à n'être que «l'idiot de la famille » plusieurs décennies après sa mort a connu, sous la plume de ses proches, bien des portraits caricaturaux au cours de son existence. Pour Maxime Du Camp, son compagnon de voyage dans tout l'ouest de la France (1847), puis en Orient (1849-1851), il sera d'abord un voyageur contradictoire, qui désire tout quitter de sa vie normande et n'a pas fait

4. Correspondance sera abrégée dès maintenant en Corr. suivi du tome et de la page.

5. Si le refus de Flaubert est bien connu devant la publication de photographies de sa personne ou devant l'illustration de ses œuvres, néanmoins il sait accorder aux images de son époque une place non négligeable au moment de créer ses propres œuvres. (Kinouchi, 2014) 
quelques kilomètres qu'il regrette déjà son pays... Pour Louise Colet, sa maîtresse, il doit devenir à jamais le Léonce d'Une Histoire de soldat (1856) et de Lui (1859), un personnage abominable auquel elle le réduit : amant ingrat, égoïste et même infidèle qui trahit les plus beaux serments d'amour et sacrifie la femme prétendument aimée à la création littéraire (Poyet et Colotte, 2021); pour les frères Goncourt, qui ne manquent pas de l'égratigner en des portraits-charges fréquents dans leur fameux Journal, Flaubert est et reste un fieffé Normand (Goncourt, 1989, 29 mars 1863), un tempérament mal dégrossi, une grosse nature aux emportements souvent pénibles, qui manque de goût $(1989,29$ octobre 1863) et dont ils décrivent ainsi l'appartement : "Cet intérieur, c'est l'homme, ses goûts et son talent; sa vraie passion est celle de ce gros Orient, il y a un fond de Barbare dans cette nature artiste ", avant de le réduire à n'être qu'un vrai " provincial outré $»^{6}$. On n'en finirait plus de citer les deux frères diaristes mais les amis les plus proches de Flaubert, de Tourgueniev à Maupassant, pour être bienveillants, eux, n'en succombent pas moins à la tentation de la caricature. Sous leur plume, Flaubert devient un Géant, un bon géant et on n'oublie pas que lui-même a revendiqué, en quelques clichés faciles, de se réclamer d'une double descendance de Vikings et de Peaux-Rouges !

On le comprend sans peine à l'énoncé de ces quelques exemples : la caricature fait l'économie de l'analyse. Elle se dispense de rendre compte de la complexité de l'homme. En réalité, la vigueur du trait n'a d'égale que son absence de justesse. En ciblant le plus visible, le plus grossier, elle fait l'impasse sur le meilleur : ce qui est caché, par discrétion et pudeur, et qui nourrit la substantifique moelle de la pensée et du caractère. Pire, la caricature résulte d'une incompréhension. Flaubert est trop peu accessible, sa personnalité trop touffue et dense pour permettre aux proches d'un jour ou même d'une décennie d'en rendre compte dans son entièreté. Mal cerné, incompris même, Flaubert subit la caricature comme une sournoise entreprise de marginalisation de sa personne. Elle invite à un isolement que l'écrivain n'en finit bientôt plus de revendiquer puisque personne n'est à sa hauteur pour le comprendre et partager ses humeurs, ses sentiments et ses pensées. De fait, quand la caricature s'impose comme la dernière image qui restera, la seule capable d'écrire le discours de la postérité, alors elle dit la bêtise d'une époque qui s'interdit la réflexion en réduisant les différences à des stéréotypes.

Et s'il n'était question que de l'homme Flaubert... Mais que n'a-t-on écrit de ses romans? La pire des ignominies tient évidemment aux discours caricaturaux portés sur son œuvre par des lecteurs qui n'y ont rien compris. Flaubert en voudra toute sa vie au procureur Pinard d'avoir réduit Madame Bovary à un roman immoral ; il souhaitera longtemps se venger du savant Guillaume Froehner qui lui intente un bien vilain

6. Les frères Goncourt développent : « Flaubert, qui est verbeux ce soir, encore plus que d'habitude et qui lance ses paradoxes non avec la légèreté de jongleur indien de Gautier, mais qui les tient péniblement en équilibre, comme un hercule de foire ou plutôt et simplement, comme un provincial outré [...]». (Goncourt, 1989, 18 janvier 1864) 
procès pour Salammbô en lui reprochant à la fois une érudition inutile et des manquements insupportables sans rien comprendre au projet littéraire de l'écrivain. Flaubert n'en finit plus de se plaindre : il se sent autorisé à déplorer l'absence d'une " poétique insciente $\aleph^{7}$, capable d'établir ses avis sur la poétique propre aux œuvres étudiées. L'écrivain s'est persuadé qu'on lit sans comprendre, et qu'on critique sans savoir. Pire, la simplification et l'exagération faisant de la caricature le mode le plus plaisant pour approcher les œuvres littéraires, Flaubert considère sa pensée, son esthétique et son style trahis par des contemporains exclusivement soucieux de défrayer la chronique (littéraire). Et l'esthète en souffre dans son exigence insatiable. L'exemple le plus marquant qui l'autorise à vouer aux gémonies une époque définitivement abandonnée à la caricature est donné par les articles de Barbey d'Aurevilly dont la violence aveugle en des charges lourdement répétées réduit l'écrivain Flaubert et son œuvre à quelques images stéréotypées qui ne rendent plus compte de sa réalité romanesque autrement plus complexe. Y a-t-il caricature plus terrible que celle d'un Flaubert en " homme à pensées rares ${ }^{8}$ ? De tant de commentaires partisans, expédiés en hâte pour remplir les colonnes d'un journal, résultats d'une lecture qui ne cherche jamais à expliquer l'œuvre, Flaubert considère qu'il n'y a rien à retirer. Ils ne servent qu'à trahir l'œuvre en des sarcasmes à oublier.

Il en va d'un sentiment tenace chez Flaubert : nul n'est accessible à personne et nulle œuvre n'est jamais comprise pour ce qu'elle est, au moins en son temps. Il faut le polissage des siècles pour échapper à la bêtise de la caricature. Par son refus du discours de la caricature, Flaubert pose les individus comme autant de monades : dans la singularité de l'individu, se dit l'impossibilité de la communion et même d'une compréhension par l'Autre. Flaubert dénonce dans le règne absolu de la caricature l'ignorance aveugle de l'Autre dans sa différence.

7. Il demande à George Sand : "Quand serait-on artiste, rien qu'artiste, mais bien artiste ? Où connaissez-vous une critique qui s'inquiète de l'œuvre en soi, d'une façon intense ? On analyse très finement le milieu où elle s'est produite et les causes qui l'ont amenées. - Mais la poétique insciente, d'où elle résulte ? Sa composition, son style ? Le point de vue de l'auteur ? Jamais ? " (Flaubert, Corr., 2 Février 1869, IV, p. 15). Ce sont aussi les premiers mots par lesquels il ouvre sa préface aux Dernières chansons de Louis Bouilhet qui traduisent le mieux son rejet de toutes les critiques qui ne sont jamais que des caricatures des œuvres lues : «On simplifierait peut-être la critique si, avant d’énoncer un jugement, on déclarait ses goûts ; car toute œuvre d'art enferme une chose particulière tenant à la personne de l'artiste et qui fait, indépendamment de l'exécution, que nous sommes séduits ou irrités. Aussi notre admiration n'est-elle complète que pour les ouvrages satisfaisant à la fois notre tempérament et notre esprit. L'oubli de cette distinction préalable est une grande cause d'injustice. »

8. Pour le romancier critique, Flaubert est un « homme à pensées rares, qui, quand il en a une, la cuit et la recuit, et non pas dans son jus, car elle n'en a pas. C'est un esprit d'une sécheresse supérieure parmi les Secs, une intelligence toute en surface, n'ayant ni sentiment, ni passion, ni enthousiasme, ni idéal, ni aperçu, ni réflexion, ni profondeur [...] ». Jules Barbey d'Aurevilly, Le Constitutionnel, 29 novembre 1869, « Variétés littéraires : L'Éducation sentimentale ». 


\section{Flaubert caricatural : une simplification méprisante? Une question de légitimité}

Si Flaubert n'aime pas être caricaturé, en revanche il ne se prive pas de caricaturer ses semblables et dans sa lutte coriace contre son époque et ses contemporains, il emploie la caricature comme une arme redoutable. Parce qu'elle est pour lui immanquablement associée à la bêtise, il la retourne contre l'Autre pour mieux en révéler son éternelle stupidité. Ainsi les personnages romanesques de l'écrivain sont-ils généralement appelés à devenir des stéréotypes. Ils sont caricaturaux au sens où leur individu est réduit à n'incarner qu'un défaut, et un seul : Emma Bovary désignera toujours la femme mal mariée et romantique, Homais le demi-habile prétendument érudit et vaniteux, Bouvard et Pécuchet des imbéciles, Arnoux le mari volage, Félicité la bonté jusqu’à la naïveté, etc. Dans son admiration pour Molière, Flaubert a réussi à le concurrencer sur le registre du personnage antonomase, après Harpagon, Tartuffe, Alceste ou Don Juan. En tant que romancier, Flaubert reproduit ce dont il souffre en tant qu'homme. Puisque ses contemporains manquent de perspicacité pour dépasser le stade des apparences et de la première impression, alors il leur offre un personnel romanesque construit selon la même logique réductrice. Emma, Homais et les autres ne parlent aux lecteurs et n'imposent le roman à la postérité que dans la mesure où le romancier accepte de leur retirer la complexité réelle de tout individu normalement constitué.

Il faudrait en effet interroger la fabrique du personnage chez l'écrivain (Guinand, 2015) pour observer comment Flaubert créant par exemple Emma Bovary procède justement par une composition caricaturale. Le point de départ? Chercher le plus grand dénominateur commun à toutes les jeunes filles qui donneront des épouses mal mariées. Il recense alors : une éducation prisonnière des murs clos d'un pensionnat, les lectures niaises qui favorisent un réel fantasmé ou encore un désir d'ascension sociale dans une rupture d'avec le passé familial. Dès lors, l'élaboration du personnage, contre les lois du réalisme, se pratique dans l'addition de toutes les causes recensées et le développement de toutes les manifestations de l'épouse romantique désillusionnée. Elle s'opère moins dans une veine réaliste - inventer un personnage avec sa singularité native, qui en fera un être commun mais unique - que dans le souci d'atteindre à un absolu indépassable : Emma est un personnage de synthèse. Il n'y aura pas de femme mariée plus insatisfaite qu'Emma après la parution de Madame Bovary. Sans peine, elle fait oublier sa sœur aînée, Madame Creton des Bourgeois de Molinchart (1855), et interdit de la surpasser à sa cadette, Fanny, l'héroïne du roman éponyme (1859) d'Ernest Feydeau. Aucune ne peut prétendre être à sa hauteur, quoique les critiques aient tâché de rapprocher les trois romans. Emma Bovary reste la figure centrale, indépassable parce que, dans son personnage, Flaubert a réuni jusqu'à la caricature toutes les raisons qui pouvaient en faire ce qu'elle devient pour l'éternité. Et si Flaubert réussit avec Madame Bovary son chef 
d'œuvre, le roman le plus lu et le plus connu de la seconde moitié du siècle, c'est que ses personnages suivants n'atteignent pas - malheureusement pour eux - au même degré de caricature.

Nous ne reviendrons pas ici sur ce qui a été bien montré par Andréa S. Landvogt dans son article "Caricature et citation : l'exemple de Madame Bovary» qui explique avec une belle pertinence comment l'écriture flaubertienne est tout entière inspirée par la caricature graphique en tant que "modèle poétologique " (2006, p. 125). Ce sur quoi nous voudrions simplement insister pour notre part, c'est la nature exacte de la démarche de Flaubert qui, en usant de la caricature, manifeste toute la sagacité de son observation. Il sait ne rater aucun des aspects constitutifs d'un personnage appelé à devenir un stéréotype sous la loi de sa plume. Là où ses contemporains le caricaturent par simplicité, c'est-à-dire par facilité, par faiblesse - ils ne sont pas capables de comprendre l'épaisseur qui est la sienne, sa richesse et même ses contradictions -, c'est au contraire par intelligence que l'écrivain Flaubert caricature des individus, grâce à la perspicacité de son regard expert et par la puissance d'une écriture qui réussit la synthèse des analyses. L'usage de la caricature ne renvoie à rien de simplificateur chez Flaubert; il ne s'agit pas de gros traits pour montrer vite et permettre de voir rapidement. Car, comme le fait remarquer Maxime Du Camp, Flaubert appartient au camp des écrivains myopes, ce qui implique que, chez lui, la caricature résulte de l'analyse répétée de tous les détails possibles, qu'elle se construise dans l'accumulation. Là où le trait du caricaturiste choisit d'aller à l'essentiel, de se réduire, de devenir même rare - quelques traits suffisent à caricaturer sous le crayon du dessinateur habile -, chez Flaubert, la caricature littéraire consiste au contraire à grossir le trait, c'est-à-dire à le dédoubler, le multiplier dans une surcharge qui vise à la totalisation. D'un côté, la volonté de l'épure ; de l'autre, le besoin d'accéder au Tout par une sorte d'écriture totale, bientôt totalitaire. Ce sont deux caricatures différentes qui se rencontrent dans l'expérience flaubertienne, la première refusée par l'homme ; l'autre, toute différente, recherchée, travaillée et même améliorée sans cesse par l'écrivain.

Construire des types, en réalité des stéréotypes, pour qu'ils deviennent les caricatures éternelles des défauts qu'ils stigmatisent. Par le choix de l'exagération, en outrant la démarche de quête de vérité qui fondait le réalisme - trop perverti en une analyse pseudo-scientifique des causes et des manifestations d'une pathologie psychologique : l'insatisfaction, la vanité, la suffisance, la bêtise, etc. -, l'écriture flaubertienne dépasse la réalité. Où trouver un curé plus vrai que Bournisien et pourtant aussi absent de la vraie vie?

La caricature s'impose, définitive, à jamais écrite pour fixer une vérité qu'elle dépasse au moment même où elle prétend en rendre compte et prémunir contre ses ravages. La caricature flaubertienne se définit en absolu de l'écriture réaliste.

Il y a longtemps, cependant, que les caricatures de Flaubert n'amusent plus que de trop rares lecteurs. Elles se donnent à lire comme un long travail, une sorte 
de produit scientifique : Flaubert invente-t-il une science de l'observation ? En tout cas, elles visent au définitif, à l'éternel. Elles apparaissent désormais moins faites pour amuser que pour révéler. On y retrouve en l'écrivain Flaubert quelque chose de Dieu dans son œuvre : la caricature du romancier Flaubert a épousé l'apparence d'un nouveau fiat lux.

Ainsi les romans de Flaubert se lisent-ils à présent comme beaucoup plus politiques - ou " engagés ", au choix - que l'écrivain l'a reconnu. Si le romancier n’affiche pas ses opinions politiques dans ses œuvres romanesques, s'il ne tient pas des chroniques dans la presse comme une tribune libre ouverte à ses propres idées, néanmoins ses personnages, dans leur constitution même, traduisent sa pensée. Car la caricature comme procédé d'écriture figure un acte militant. Elle exprime le mépris d'un type de personne. Le personnage-charge traduit un rejet, résultat tout à la fois du pessimisme de l'écrivain, de sa misanthropie et même de son nihilisme, sur lesquels nous allons revenir pour terminer.

\section{Nihilisme, politique et modernité : I'« hénaurrrme »}

Chez Flaubert, la caricature renvoie volontiers à l'« hénaurrrme ». L'exagération ou l'hyperbole amusent l'écrivain qui sait leur puissance déstabilisatrice au cœur d'une société bourgeoise face à laquelle il oppose le projet, maintes fois répétées, d'un renversement plus ou moins anarchiste, voire nihiliste. Déformer, parodier, dégrader : tel est le pouvoir de l'écrivain qui trouve dans la caricature le moyen simple et réconfortant d'afficher sa pensée politique. Celle-ci n'est jamais que le fruit d'une précaution individuelle : ne pas avoir d'illusions pour ne pas connaître de désillusions. En caricaturant les gens et les opinions, dans sa vie et dans son ouvre, Flaubert participe d'une déconstruction généralisée de la société bourgeoise. À les réduire à une image par trop simplificatrice, les hommes finissent par être niés; et les idées s'effondrent dans le ridicule qui les frappe. Tout s'amenuise jusqu'à une disparition définitive. La caricature ne cesse de répéter que rien ne vaut. Ainsi occupé à la mise en place d'une politique d'anéantissement des valeurs communément partagées, Flaubert concourt à l'affirmation du pouvoir de l'écrivain, juste avant l'avènement de l'autotélisme de l'art, autrement dit la victoire de la modernité9.

Flaubert a osé, dans l'intimité, créer des êtres loufoques comme le Révérend Père Cruchard (Flaubert, 2005) ou, plus tôt, et de manière plus collégiale, la figure

9. En conclusion de son lumineux article "Penser le rire au XIX ${ }^{e}$ siècle à travers les histoires de la caricature », Michela Lo Feudo fait remarquer : "En fonction du développement d'une caricature considérée comme un moyen nouveau d'expression et de communication, on pourrait pousser la réflexion jusqu'à penser que l'image satirique, porteuse d'un rire oscillant entre terreur et hilarité, offre un véritable modèle pour les écrivains du XIX ${ }^{e}$ siècle. » (Lo Feudo, 2013) 
du Garçon ${ }^{10}$. À travers ses figures cocasses, affranchies des contraintes de la littérature sérieuse vouée à l'édition, il a proposé des personnages libertaires qui donnent une vraisemblance à l'impossible : le vacillement des bienséances de l'époque en même temps que la promotion d'une nouvelle manière d'être, libérée, spontanée, indépendante. Or, le romancier a poursuivi l'aventure, plus sérieusement mais sans avoir le temps d’en assumer les conséquences éditoriales, avec le Dictionnaire des idées reçues. Là encore, il s'est agi de caricaturer le contemporain par la recension de toutes les bêtises proférées et adoptées par l'époque. Fort d'un pareil texte, Flaubert continue de mettre en place une politique de la table rase ; il réduit à néant tout ce qui se pense, dans un arasement vengeur. Que cela puisse ne pas plaire, il s'en doute tant la société de la Troisième République lui a donné un avant-goût de la réception qui l'attendrait avec celle, calamiteuse, que ses contemporains avaient réservée en 1874 à sa pièce, Le Candidat. En réalité, l'œuvre de Flaubert rappelle une question fondamentale en pleine consécration de l'écriture réaliste : à quoi sert la caricature?

Pour sa part, le romancier use de la caricature pour renverser les valeurs, inviter à renoncer aux codes jugés désuets, inciter à démolir les statues. En se permettant toutes les audaces, en ne s'interdisant aucune privauté, en se démultipliant - bientôt tout est sujet à l'exercice impitoyable de la caricature -, Flaubert peut scandaliser (Poyet, 2021) et, ce faisant, fragiliser une société condamnée à mesurer combien une esthétique de l'outrance se confond avec une éthique de la révolte. En privé, Flaubert n'hésite pas à avouer : «L'excès m'a toujours attiré, quel qu'il soit. » (Flaubert, Corr., à Louise Colet, 10 octobre 1846, I, p. 383) Cependant, l'exercice qui consiste à se marginaliser sans cesse, et qu'il pratique tout au long de son existence, ne fonctionne pas comme une planche de salut. L'homme Flaubert y puise au contraire de quoi nourrir sa désespérance qui s'exprime d'abord à travers une répétition forcée de sa misanthropie: « Je déteste fort mes semblables et ne me sens pas leur semblable. - C'est peut-être un monstrueux orgueil, mais le diable m'emporte si je ne me sens pas aussi sympathique pour les poux qui rongent un gueux que pour le gueux. Je suis sûr d'ailleurs que les hommes ne sont pas plus frères les uns aux autres que les feuilles des bois ne sont pareilles. » (Flaubert, Corr., à Louise Colet, 26 mai 1853, II, p. 335)

La Correspondance de l'écrivain montre un homme désireux de s'affirmer dans une singularité qui le hausse au-dessus de ses contemporains. N'est-ce pas lui qui affirme, un peu imbu de lui-même : «Mon pauvre Bouilhet me disait souvent : "Il n'y a pas d'homme plus moral ni qui aime l'immoralité plus que toi : une infamie te réjouit". Il y a du vrai là-dedans. Est-ce un effet de mon orgueil ? ou par une certaine perversité ? " Mais l'expression de ce qui pourrait n'être qu'une folle prétention renvoie toujours à la même lente et sourde désespérance. Le rire-condamnation,

10. Jean Bruneau explique : «Le "Garçon" est une création collective de Flaubert et de ses camarades. Il représente à la fois le bourgeois Louis-philippard et le farceur qui se moque des bourgeois. Flaubert et ses amis pouvaient satisfaire, par son intermédiaire, aussi bien leurs aspirations idéalistes que leur sens aigu de la satire et de la farce. » (Jean Bruneau, in Flaubert, Corr., II, p. 852) 
celui qui prononce la sentence venue s'abattre sur la cohorte gigantesque des idiots, se transforme forcément en éclat de colère : la caricature n'est que le rire du vengeur. Elle se finalise toujours sur une disparition prochaine de la parole vengeresse et de celui qui la prononce, usé par une opposition trop fournie, comme l'explique Philippe Dufour :

L'ironie de Flaubert n'est pas celle de Voltaire. Elle n'a pas une vérité à proposer en lieu et place des mensonges qu'elle dénonce. Elle est un plaisir amer. Elle sollicite la complicité d'un lecteur à qui elle n'a pas de secret à révéler. Elle demeure une parole avortée. Elle se tient en marge de la parole de masse, mais est voisine du silence. (Dufour, 1993, p. 185)

Flaubert sait tous les risques de la caricature, lui qui annonce : "Je crois qu'on n’a pas encore tenté le comique d'idées? il est possible que je m'y noie, mais si je m’en tire, le globe terrestre ne sera pas digne de me porter. » (Flaubert, Corr., à Edma Roger des Genettes, 2 avril 1877, V, p. 213)

Mais revenons à la réalité de la caricature dans la seconde moitié du siècle. Si Flaubert n'a probablement pas lu son confrère Champfleury, et ses cinq volumes d'histoire de la caricature ${ }^{11}$, a-t-il eu connaissance des articles de Baudelaire sur le rire ${ }^{12}$ et les caricaturistes ${ }^{13}$ ? Rien n'est moins sûr et pourtant les analyses du poète renseignent avec pertinence sur le rapport à la caricature de Flaubert. Baudelaire considère que le " rire est l'explosion perpétuelle de [1]a colère et de [1]a souffrance. Il est, qu'on me comprenne bien, la résultante de sa double nature contradictoire, qui est infiniment grande relative à l'homme, infiniment vile et basse relativement au Vrai et au Juste absolus. » (p. 531) Et le poète de rappeler encore qu'il y a « au fond de la pensée du rieur un certain orgueil inconscient » (p. 531) : il insiste sur la dimension « essentiellement contradictoire [du rire], c'est-à-dire qu'il est à la fois signe d'une grandeur infinie et d'une misère infinie, misère infinie relativement à l'Être absolu dont il possède la conception, grandeur infinie relativement aux animaux. » (p. 532)

Il va de soi que le rire de Flaubert tel qu'il s'exprime dans l'écriture de la caricature s'assimile en tous points à la définition posée par son contemporain. En termes familiers, Flaubert rit jaune, il ne sait pas faire autre chose. Si la caricature de l'humanité revient à mesurer avec perspicacité tous ses défauts, le romancier ne peut omettre qu'il appartient aussi à cette humanité risible. Et de se sentir forcément risible, à son tour... Le rieur doit rire de lui-même.

11. Histoire de la caricature antique et Histoire de la caricature moderne (Dentu, 1865), Histoire de la caricature au Moyen Âge (1872), Histoire de la caricature sous la République, l'Empire et la Restauration (Dentu, 1877) et Histoire de la caricature sous la Réforme et la Ligue (Dentu, 1880).

12. «De l'essence du rire" paraît pour la première fois en 1855, dans Le Portefeuille.

13. «Quelques caricaturistes français » est publié pour la première fois le ler octobre 1857 dans Le Présent ; "Quelques caricaturistes étrangers » paraît pour la première fois, le 15 octobre 1857, toujours dans Le Présent. (Baudelaire, 1976) 
Mais le lecteur s'extasie encore devant la justesse du jugement de Baudelaire quand, définissant l'art de la caricature chez Daumier, il renvoie à la fois à « la certitude » de l'artiste, à « un talent d'observation tellement sûr », à « la logique du savant transportée dans un art léger, fugace, qui a contre lui la mobilité même de la vie ", à la capacité d'aller « droit au but ». Comme chez Molière, «l'idée se dégage d'emblée. On regarde, on a compris. » (p. 556), estime Baudelaire admiratif. Or, la technique de Daumier n'est pas sans hériter de la ligne de Léonard de Vinci : ses caricatures, le génial italien « les a faites en savant, en géomètre, en professeur d'histoire naturelle. Il n'a eu garde d'omettre la moindre verrue, le plus petit poil.» (p. 570) L'art de Vinci ou l'art de Daumier, n'est-ce pas en fin de compte l'art de Flaubert caricaturiste qui tient tout entier dans la volonté d'une observation exhaustive pour mieux rendre implacable la critique et indiscutable la portée de la caricature?

Quand le réalisme de Flaubert se fait paradoxalement pratique de la caricature, il renvoie à l'engagement d'un romancier qui ne supporte plus la société de son époque et qui, seétant interdit par sa poétique le droit d'exprimer son opinion, n'en désire pas moins rendre compte du réel de telle sorte que nul ne puisse plus s'en dire innocent. Sartre s'enfonçait dans l'erreur la plus complète à tenir Flaubert (et les Goncourt) dans le texte manifeste de 1945 des Temps modernes " pour responsables de la répression qui suivit la Commune parce qu'ils n'ont pas écrit une ligne pour l'empêcher. Ce n'était pas leur affaire, dira-t-on. Mais le procès de Calas, était-ce l'affaire de Voltaire ? La condamnation de Dreyfus, était-ce l'affaire de Zola ? L'administration du Congo, était-ce l'affaire de Gide ? Chacun de ces auteurs, en une circonstance particulière de sa vie, a mesuré sa responsabilité d’écrivain. »(Sartre, 1945) Au contraire, bien au contraire...

Flaubert n'a pas cessé d'écrire contre son temps et ses erreurs, à sa manière. Frédéric Moreau, Arnoux et son épouse, Rosanette, la Vatnaz, Pellerin ou Hussonnet, que sont-ils d'autres que des caricatures d'hommes et de femmes composées justement pour éviter les hommes et les femmes de la Commune?

\section{RÉFÉRENCES}

Baudelaire, Ch. (1976). Euvres complètes. T. 2. C. Pichois (éd.). (p. 525-543, p. 544-563 et p. 564-574). Paris : Gallimard.

Dufour, P. (1993). Flaubert ou la parole du Pignouf. Vincennes : Presses Universitaires de Vincennes.

Flaubert, G. (1973-2007). Correspondance. T. I (1973), T. II (1980), T. III (1991), T. IV (1998), T. V (2007). J. Bruneau - et Y. Leclerc pour le tome V (éd.). Paris : Gallimard.

Flaubert, G. (2005). Vie et travaux du R. P. Cruchard et autres inédits. M. Desportes et Y. Leclerc (éds.). Mont-Saint-Aignan : Publications des Universités de Rouen et du Havre.

Goncourt, E. et J. de. (1989). Journal. Mémoires de la vie littéraire. R. Ricatte (éd.). Paris : Robert Laffont. 
Guinand, C. (2015). La caricature littéraire : L'Éducation sentimentale de Flaubert. Quêtes littéraires, 5, 65-77. https://doi.org/10.31743/q1.239

Kinouchi, T. (2014). La mémoire des images dans L'Éducation sentimentale, Flaubert [En ligne], 11 | 2014, mis en ligne le 17 octobre 2014. http://journals.openedition.org/flaubert/2256

Landvogt, A. S. (2006). Caricature et citation : l'exemple de Madame Bovary. Dans J. Bem et U. Dethloff avec la coll. d'A. Barjonet (textes réunis par), Nouvelles lectures de Flaubert. Recherches allemandes (p. 110-129). Tübingen : Gunter Narr Verlag.

Lo Feudo, M. (2013). Penser le rire au XIX ${ }^{\mathrm{e}}$ siècle à travers les histoires de la caricature. Dans A. Vaillant et R. de Villeneuve (dirs.), Le rire moderne (p. 332-333). Paris : Presses Universitaires de Paris Ouest.

Poyet, T. (2021). Le scandaleux sans scandale. Au bonheur du tout-dire chez Flaubert épistolier. Dans É. Le Calvez (dir.). Flaubert et le scandale. Paris : Éditions Passages. (sous presse).

Poyet, T. et Colotte, F. (2021). Flaubert ou l'ouvre muse. Paris : Kimé, (chapitre 3 : «Flaubert personnage romanesque »). (sous presse).

Sartre, J.-P. (1945). Présentation. Les Temps Modernes, $\mathrm{n}^{\circ} 1,1^{\mathrm{er}}$ octobre 1945.

RÉSUMÉ : Quelquefois caricaturé par ses contemporains, Flaubert suscite les réactions dans l'outrance, lui qui ose dans sa Correspondance abonder dans la caricature selon ses humeurs et ses opinions en excellant dans l'hyperbole et l'emphase. Un mot de son cru le résume : "hénaurrrme ». Au demeurant, ses personnages les plus mémorables sont des caricatures : de l'amour avec Emma, de la bêtise avec Bouvard et Pécuchet, de la bonté avec Félicité d'Un Cour simple... Dans la fabrique flaubertienne, l'incarnation est intrinsèquement liée à la déformation, elle-même dégradée en parodie. Car la caricature chez Flaubert est partie prenante d'une pensée nihiliste : le romancier participe d'une déconstruction généralisée de la société bourgeoise par une caricature évidemment politique même si l'écrivain se défend d'utiliser l'œuvre comme une tribune, au nom de l'autotélisme de l'art. Au fond, c'est la modernité de Flaubert qui justifie le goût et l'usage de la caricature.

Mots-clés : Flaubert, réalisme, engagement, modernité littéraire

\section{Flaubert and caricature: the demand for modernity}

ABSTRACT: Caricatured at times by his contemporaries, Flaubert provokes extreme reactions. In his Correspondence, he dares to present a plethora of caricatures which suit his moods and his opinions, excelling in hyperbole and emphasis. A word of his own summed it up: " hénaurrme ». Besides, his most memorable characters are caricatures : Emma - of love, Bouvard and Pécuchet - of stupidity, Félicité of Un cour simple - of kindness. In Flaubert's factory, the incarnation is intrinsically linked to deformation, itself degraded into a parody. Because the caricature in Flaubert is part of a nihilistic thought: the novelist participates in a general deconstruction of bour- 
geois society by an obviously political caricature even if the writer refrains from using the work as a platform, in the name of "autotelism" of art. Basically, it is Flaubert's modernity that justifies his taste and the use of caricature.

Keywords: Flaubert, realism, writer engagement, literary modernity 